\section{PTH-113 CHANGE IN AWARENESS OF GLUTEN RELATED DISORDERS AMONGST CHEFS AND THE GENERAL PUBLIC IN THE UNITED KINGDOM: A 10 YEAR FOLLOW-ON STUDY}

I Aziz* M Karajeh, J Zilkha, E Tubman, C Fowles, DS Sanders. Department of Gastroenterology, Royal Hallamshire Hospital, Sheffield Teaching Hospitals, Sheffield, UK

10.1136/gutjnl-2014-307263.559

Introduction For individuals with gluten-related disorders (GRD) eating out has traditionally been difficult, and socially impacting, due to concern over the lack of public awareness regarding GRD and a gluten-free diet (GFD). However, the recent rise in media coverage highlighting these conditions may have altered knowledge amongst community members.

Aims To assess whether there has been a change in awareness of GRD, and a GFD, amongst the general public and chefs over a ten year period.

Methods A face-to-face questionnaire survey about coeliac disease (CD) and gluten sensitivity (GS) was performed on the general public and chefs based in Sheffield, United Kingdom. The assessment was first conducted in 2003 and repeated in 2013. Chefs were also asked about their workplace (takeaway or restaurant) and whether or not they had formal qualifications. Additional questions for the 2013 cohort included correct recognition of the cross-grain symbol to identify gluten-free products and whether they displayed a notice/sign for gluten-free products.

Results Public survey: 513 public members in year 2003 (mean age 49.2 , 62\% female) were compared to 575 public members in year 2013 (mean age 37.8, 57\% female). Adjusting for age and sex, there was a significant rise in the awareness of GRD from the years 2003 to 2013 ; CD (44.2 to $74.4 \%$, OR 3.94 [CI: 2.99-5.19]) and GS (58.2 to 89\%, OR 7.09 [CI: 5-9.98]), p value $<0.0001$.

Chef Survey: 322 chefs in year 2003 (mean age 37.6, 15.2\% female, qualified $51.2 \%$, restaurant chefs $50 \%$ ) were compared to 265 chefs in year 2013 (mean age 27.1, 38.1\% female, qualified $93.2 \%$, restaurant chefs 83\%), $\mathrm{p}<0.0001$. Adjusting for age, sex, workplace and qualifications, there was a significant rise in the awareness of GRD from the years 2003 to 2013; CD (17.1 to $78.1 \%$, OR 12.5 [CI: $7.9-19.6]$ ) and GS (9.3 to $87.5 \%$, OR 65.7 CI: [35.4-122]), $\mathrm{p}<0.001$.

Whereas in 2003 the public were significantly more aware of GRD than chefs, by 2013 there was a similar prevalence of awareness in both groups. In addition, the correct recognition of the gluten-free symbol was $44 \%$ for the public and $40 \%$ for chefs (p 0.28). Furthermore, in the year 2013, 41\% of restaurants and $27 \%$ of takeaways displayed selling gluten-free products (p 0.07).

Conclusion There has been a dramatic rise in both the public and chefs awareness of GRD. This suggests that individuals with GRD can take greater confidence discussing and ordering a GFD whilst eating out.

Disclosure of Interest None Declared.

\section{PTH-114 ASSESSING OSTEOPOROSIS IN COELIAC DISEASE: IS THE WHO FRAX TOOL A GOOD SCREENER?}

1J Weightman*, ${ }^{2} \mathrm{M}$ Bridges, ${ }^{1} \mathrm{~A}$ Dhar. ${ }^{1}$ Gastroenterology, County Durham and Darlington NHS Foundation Trust, Co. Durham, UK; ${ }^{2}$ Rheumatology, County Durham and Darlington NHS Foundation Trust, Co. Durham, UK

10.1136/gutjnl-2014-307263.560
Introduction Osteoporosis is a well-recognised complication of intestinal malabsorption related to Coeliac Disease. It is diagnosed by dual energy x-ray absorptiometry (DXA). While referral for DXA scanning in other conditions is widely based on 10year fracture risk calculated by the FRAX tool designed by the WHO, the 2007 BSG guidelines advise screening patients with a higher risk of osteoporosis with a DXA scan, irrespective of 10year fracture risk as calculated by FRAX.

Methods Aim: We aimed to establish whether the FRAX and linked NOGG tool was a good screener to determine the need for DXA scan in patients with coeliac disease who are at risk of osteoporosis.

Methods: We used the Hospital Nuclear Medicine database to retrieve the records of 50 patients with a diagnosis of coeliac disease who had been investigated with a DXA scan to assess osteoporosis. Using their medical records, we then calculated their FRAX score for risk of major osteoporotic fracture and hip fracture, and the corresponding NOGG guidance. A 10-year fracture risk of $10 \%$ or the linked NOGG guidance was considered to be significant to have a DXA scan.

Results Of the 50 patients with Coeliac disease who had DXA scans, 10 had osteoporosis and 40 had either a normal result or osteopaenia. Of these 10 cases, 9 would have also been referred on the basis of a calculated FRAX score and only one case would have been falsely reassured. Of the 40 cases with either a normal DXA scan or osteopaenia, 31 would have been referred for DXA on the basis of the FRAX score, resulting in an unnecessary test. We concluded that a positive FRAX score does not accurately predict osteoporosis in Coeliac disease. The positive predictive value of the FRAX tool to detect osteoporosis in Coeliac disease is low at $22.5 \%$; however the negative predictive value is high, 90\%.

Conclusion The use of FRAX to identify patients with Coeliac disease at risk of osteoporosis has a high negative predictive value. It therefore has merit as a screening tool but has little value as a diagnostic test. Although the sample size was too small to show statistical significance, we suggest that FRAX tool could potentially be adopted as a screener in the context of celiac disease to prevent unnecessary DXA scanning. A osteoporosis risk of $<10 \%$ or a NOGG guidance of reassurance is likely to be associated with a normal DXA scan. Further large studies are needed to validate this hypothesis and also to determine cost benefit of a FRAX driven strategy for osteoporosis in Coeliac Disease.

Disclosure of Interest J. Weightman: None Declared, M. Bridges: None Declared, A. Dhar Consultant for: Honoraria for advisory roles ot the Pharmaceutical Industry, Speaker bureau with: Warner Chilcott UK, Falk Pharma UK, Almirall, Ferring Pharma UK, Astellas UK.

\section{PTH-115 PREVALENCE RATES AND RISK FACTORS FOR OSTEOPOROSIS IN PATIENTS WITH COELIAC DISEASE}

1J Schembri*, ${ }^{2} \mathrm{P}$ Torpiano, ${ }^{1} \mathrm{~N}$ Azzopardi, ${ }^{1} \mathrm{M}$ Vassallo, ${ }^{1} \mathrm{P}$ Ellul. ${ }^{1}$ Department of Gastroenterology, Mater Dei Hospital, Msida, Malta; ${ }^{2}$ Department of Paediatrics, Mater Dei Hospital, Msida, Malta

\subsection{6/gutjnl-2014-307263.561}

Introduction The optimal timing for performing a baseline bone mineral density (BMD) in Coeliac disease (CD) patients is controversial. European guidelines published in 1998 recommended a baseline BMD at diagnosis. One study in 2005 demonstrated a low incidence of BMD abnormalities amongst 
patients with $\mathrm{CD}^{1}$ and the British Society of Gastroenterology guidelines state that DEXA should only be done after introduction of a gluten-free diet on the subgroups of patients in whom the risk of osteoporotic fracture is high $^{2}$. This was however followed up by a guidance document in 2010 stating that BMD assessment should always be performed at diagnosis. Meanwhile the American guidelines suggest testing for vitamin and micronutrient deficiencies ${ }^{3}$. The aims of this study were to determine, the prevalence of osteopenia and osteoporosis among patients who are newly diagnosed with $\mathrm{CD}$, and any risk factors which would increase patients' risk of osteopenia and osteoporosis.

Methods We carried out a prospective cohort study, where newly diagnosed CD patients were recruited. DEXA scanning was done at diagnosis. Data with regards to smoking, BMD and histology was entered into a database and analysed using SPSS software package.

Results 137 patients with a histological diagnosis of CD were recruited. $76.6 \%$ were females. Mean age at diagnosis was 37.1 years (95\% CI: \pm 3.19 years). $21.9 \%(\mathrm{n}=30)$ of patients were osteoporotic and another 51.1\% $(\mathrm{n}=72)$ were osteopenic at diagnosis. A total of $14.9 \%(\mathrm{n}=17)$ had a previous history of fracture/s prior to diagnosis. Osteoporosis at the spine was significantly associated with the female gender $(\mathrm{p}=0.04)$ and with an older age at diagnosis $(50.3$ years $p=0.01$; 95\% CI: \pm 6.6 years). Patients with Marsh $3 \mathrm{c}$ disease at diagnosis were also more likely to have an abnormal BMD at the spine than patients with Marsh 3a or 3b $(p=0.04)$. Mean BMI between osteopenic $\left(24.15 \mathrm{~kg} / \mathrm{m}^{2}\right.$ 95\% CI: \pm 1.29$)$ and osteoporotic $\left(23.37 \mathrm{~kg} / \mathrm{m}^{2}\right.$ 95\% CI: \pm 2.81$)$ patients was slightly different but not statistically significant $(\mathrm{p}=0.07)$.

Conclusion This data demonstrates a high rate of osteopenia and osteoporosis among CD patients at diagnosis. DEXA scanning should therefore be considered at diagnosis. This is of greater importance in female patients diagnosed at or above the age of 50 years and with Marsh $3 \mathrm{c}$ disease.

\section{REFERENCES}

1 Lewis NR, Scott BB. Should patients with coeliac disease have their bone mineral density measured? Eur J Gastroenterol Hepatol 2005 Oct:17(10):1065-70

2 NR Lewis, BB Scott. Guidelines for osteoporosis in inflammatory bowel disease and coeliac disease. British Society of Gastroenterology June 2007

3 American Gastroenterological Association medical position statement: Guidelines on osteoporosis in gastrointestinal diseases. Gastroenterology - March 2003;124 (3): 791-794)

Disclosure of Interest None Declared.

\section{PTH-116 POINT OF CARE TESTING FOR ADULT COELIAC DISEASE: A POTENTIAL ROLE IN ENDOSCOPY}

${ }^{1} \mathrm{~S}$ Wong*, ${ }^{1} \mathrm{PD}$ Mooney, ${ }^{1} \mathrm{M}$ Kurien, ${ }^{1} \mathrm{AJ}$ Johnston, ${ }^{1} \mathrm{~A}$ Avgerinos, ${ }^{2} \mathrm{M}$ Hadjivassiliou, ${ }^{1}$ DS Sanders. ${ }^{1}$ Regional GI and Liver Unit, Royal Hallamshire Hospital, Sheffield, UK; ${ }^{2}$ Neurology, Royal Hallamshire Hospital, Sheffield, UK

\subsection{6/gutjnl-2014-307263.562}

Introduction Endoscopic markers of coeliac disease (CD) lack sensitivity; therefore many centres take routine duodenal biopsies or have a low threshold for biopsy, ensuring high detection rates. Newly available, point of care tests (POCT) provide rapid findings unlike conventional serological markers, potentially reducing the need for duodenal biopsies. This study evaluates a new POCT (Simtomax) which detects IgA and IgG deamidated gliadin peptide (DGP) with comparisons made to conventional serological markers and histology.
Methods Patients referred for a gastroscopy to a specialist CD list were prospectively recruited between March and November 2013. Patients were excluded if they were on a gluten free diet at the time of the test or if they had previously been diagnosed with seronegative villous atrophy. All patients had a duodenal biopsy as the gold standard for detecting CD. Concurrently serological testing for IgA tissue transglutaminase (TTG), endomysial antibody (EMA), total immunoglobulin A level and the DGP based rapid test was performed. Sensitivity, specificity, positive predictive (PPV) and negative predictive values (NPV) were calculated.

Results 354 patients met the inclusion criteria $(45.8 \%$ male mean age $53.3+18.5)$. Of these, $52(14.7 \% 11.2-18.9)$ had newly diagnosed CD and 302 were controls with a normal duodenal biopsy. The sensitivity, specificity, PPV and NPV for the POCT were 94, 83, 49 and 99\% respectively. This compares with results for TTG of 92, 88, 57, 99 and EMA of 88, 97, 85, and 98\% respectively. In a second cohort, 43 patients with known $\mathrm{CD}$ for re-assessment were recruited (20.9\% male, mean age 49.4 +16.6). 16 (37\% 23 -53) of these 16 patients $(37 \%)$ had persistent villous atrophy despite a gluten free diet. POCT compared to histology showed sensitivity of $88 \%$ and specificity $41 \%$. tTG showed sensitivity and specificity of 63 and 70\% respectively and EMA 56 and 78\% respectively. However agreement between histology and POCT was poor with concordance between results in only $60 \%$ $(\kappa=0.274)$. tTG and EMA were marginally better with $\kappa=0.321$ and $\kappa=0.345$ respectively.

Conclusion This is the first study to prospectively demonstrate the value of a novel POCT for adult CD in endoscopy compared to the gold standard of histology. The sensitivity and specificity of the POCT is comparable to conventional serology. Simtomax could be used to appropriately identify patients requiring a duodenal biopsy within the endoscopic setting. This strategy may be cost effective by reducing the number of routine duodenal biopsies taken. Further work is required to clarify the role of POCT for the assessment of histological remission in patients with known CD.

Disclosure of Interest None Declared.

\section{PTH-117 THYMIC STROMAL LYMPHOPOIETIN IS PRIMARILY REDUCED IN REFRACTORY COELIAC DISEASE DUODENAL MUCOSA}

${ }^{1} \mathrm{P}$ Biancheri ${ }^{*},{ }^{2} \mathrm{~A}$ Di Sabatino, ${ }^{3} \mathrm{G}$ Fornasa, ${ }^{4} \mathrm{C}$ Papadia, ${ }^{4} \mathrm{R}$ Shidrawi, ${ }^{4} \mathrm{E}$ Woods, ${ }^{5} \mathrm{~A}$ Forbes, ${ }^{3} \mathrm{M}$ Rescigno, ${ }^{2} \mathrm{GR}$ Corazza, ${ }^{1} \mathrm{TT}$ MacDonald. 'Centre for Immunology and Infectious Disease, Barts and the London School of Medicine and Dentistry, London, UK; ${ }^{2}$ First Department of Medicine, Fondazione IRCCS Policlinico S. Matteo, University of Pavia, Pavia, Italy; ${ }^{3}$ Department of Experimental Oncology, European Institute of Oncology, Milan, Italy; ${ }^{4}$ Academic Department of Medical and Surgical Gastroenterology, Homerton University Hospital, London, UK; ${ }^{5}$ Norwich Medical School, Norwich, UK

\subsection{6/gutjnl-2014-307263.563}

Introduction Thymic stromal lymphopoietin (TSLP), a cytokine released by enterocytes and gut dendritic cells, promotes the development of Foxp3 + regulatory T cells and at the same time inhibits the development of pro-inflammatory $\mathrm{T}$ helper (Th)1 and Th17 cells. While mucosal TSLP expression is down-regulated in untreated coeliac disease (CD), its levels are unknown in refractory $\mathrm{CD}(\mathrm{RCD})$, in which the transformation of aberrant intraepithelial $\mathrm{T}$ cells predisposes to the emergence of enteropathy-associated $\mathrm{T}$ cell lymphoma. Therefore, we evaluated the 